\title{
Novel Regulation of Parkin Function through c-Abl-Mediated Tyrosine Phosphorylation: Implications for Parkinson's Disease
}

\author{
Syed Z. Imam, ${ }^{1,2,3,4}$ Qing Zhou, ${ }^{1}$ Ayako Yamamoto, ${ }^{5}$ Anthony J. Valente, ${ }^{1}$ Syed F. Ali, ${ }^{6}$ Mona Bains, ${ }^{2}$ James L. Roberts, ${ }^{2,3}$ \\ Philipp J. Kahle, ${ }^{5}$ Robert A. Clark, ${ }^{1,3,4}$ and Senlin $\mathrm{Li}^{1,2,3,4}$ \\ Departments of ${ }^{1}$ Medicine and ${ }^{2}$ Pharmacology and the ${ }^{3}$ Barshop Institute for Longevity and Aging Studies, University of Texas Health Science Center, San \\ Antonio, Texas 78229, ${ }^{4}$ South Texas Veterans Health Care System, San Antonio, Texas 78229, ${ }^{5}$ Laboratory of Functional Neurogenetics, Department of \\ Neurodegeneration, Hertie Institute for Clinical Brain Research, University Clinics Tübingen, 72076 Tübingen, Germany, and ${ }^{\circledR}$ Neurochemistry Laboratory, \\ Division of Neurotoxicology, National Center for Toxicological Research/United States Food and Drug Administration, Jefferson, Arkansas 72079
}

Mutations in parkin, an E3 ubiquitin ligase, are the most common cause of autosomal-recessive Parkinson's disease (PD). Here, we show that the stress-signaling non-receptor tyrosine kinase c-Abl links parkin to sporadic forms of PD via tyrosine phosphorylation. Under oxidative and dopaminergic stress, c-Abl was activated in cultured neuronal cells and in striatum of adult C57BL/6 mice. Activated c-Abl was found in the striatum of PD patients. Concomitantly, parkin was tyrosine-phosphorylated, causing loss of its ubiquitin ligase and cytoprotective activities, and the accumulation of parkin substrates, AIMP2 (aminoacyl tRNA synthetase complex-interacting multifunctional protein 2) (p38/JTV-1) and FBP-1.STI-571, a selective c-Abl inhibitor, prevented tyrosine phosphorylation of parkin and restored its $\mathrm{E} 3$ ligase activity and cytoprotective function both in vitro and in vivo. Our results suggest that tyrosine phosphorylation of parkin by c-Abl is a major post-translational modification that leads to loss of parkin function and disease progression in sporadic PD. Moreover, inhibition of c-Abl offers new therapeutic opportunities for blocking PD progression.

\section{Introduction}

Parkinson's disease (PD), is a neurodegenerative movement disorder characterized pathologically by progressive loss of midbrain dopaminergic neurons (Olanow and Tatton, 1999) and protein inclusions designated Lewy bodies and Lewy neurites (Braak et al., 2003). Although $>90 \%$ of PD cases occur sporadically and are thought to be caused, in part, by oxidative stress and mitochondrial dysfunction (Zhang et al., 2000a), study of genetic mutations has provided great insight into molecular mechanisms of PD (Dawson and Dawson, 2003; Moore et al., 2005). Mutations in parkin, which encodes E3 ubiquitin ligase (Shimura et al.,

\footnotetext{
Received April 10, 2010; revised Aug. 30, 2010; accepted 0ct. 23, 2010.

This work was supported by grants from the US National Institutes of Health, Department of Veterans Affairs, Michael J. Fox Foundation, Parkinson's Disease Foundation, San Antonio Area Foundation, American Parkinson Disease Association, Executive Research Council of University of Texas Health Science Center, San Antonio (UTHSCSA), the German National Genome Research Network (NGFN-2), and the European Union (N)EUROPARK consortium. The technical assistance of Xuyean Liao, Doran Pearson, Lora Judge, Heather Dudley, and Wuqiong Ma is acknowledged. We thank F. Fiesel (Hertie Institute, Tübingen, Germany) for helpful suggestions, and S. T. Weintraub and K. Hakala for MS analyses (UTHSCSA Institutional Mass Spectrometry Laboratory). Parkin plasmids and constructs, antibodies to AIMP2, and postmortem human samples were kind gifts from Ted M. Dawson (Johns Hopkins University, Baltimore, MD). We also thank Dr. Dawson's lab for processing of some human samples. c-Abl and Arg constructs were kind gifts from B. Li (Institute of Molecular and Cell Biology, Singapore), D. Kufe (Dana-Farber Cancer Institute, Harvard Medical School, Cambridge, MA), and Z. M. Yuan (Harvard School of Public Health). Recombinant tyrosine kinase fragment of c-Abl was a kind gift from M. Seeliger and J. Kuriyan (Howard Hughes Medical Institute, University of California, Berkeley, CA). FBP-1 CDNA was kindly provided by D. Levens (National Cancer Institute, National Institutes of Health, Bethesda, MD). Lenti-shRNA to parkin was kindly provided by P. Aebischer and R. Zuffrey (École Polytechnique Fédérale Lausanne, Lausanne, Switzerland). STI-571 was provided by Novartis Pharma.

This article is freely available online through the $J$ Neurosci Open Choice option.

Correspondence should be addressed to either of the following: Syed Z. Imam or Senlin Li, University of Texas Health Science Center, 7703 Floyd Curl Drive, San Antonio, TX 78229. E-mail: imam@uthscsa.edu or lis1@ uthscsa.edu.

DOI:10.1523/JNEUROSCI.1833-10.2011

Copyright $\odot 2011$ the authors $\quad 0270-6474 / 11 / 310157-07 \$ 15.00 / 0$
}

2000; Zhang et al., 2000b), are among the most common causes of hereditary PD (Kitada et al., 1998). These mutations are thought to impair parkin activity through direct loss of function, diminished parkin solubility, or impaired degradation of substrates (Sriram et al., 2005; Matsuda et al., 2006). Numerous putative parkin substrates have been described, and failure of parkin to ubiquitinate some of these substrates may play important role in dopaminergic neurodegeneration (von Coelln et al., 2004). Particularly, aminoacyl-tRNA synthetase complex-interacting multifunctional protein type 2 (AIMP2) (p38/JTV-1) (Corti et al., 2003) and far upstream element-binding protein-1 (FBP-1) seem to be authentic parkin substrates, as they accumulate in parkin-deficient mice and in brain tissue of patients with hereditary PD (Ko et al., 2005, 2006). Furthermore, AIMP2 is selectively toxic to dopaminergic neurons (Ko et al., 2006). Other substrates may also play a role in PD (von Coelln, 2004; Moore et al., 2005). Oxidative, nitrative, nitrosative, and dopaminergic stress are thought to impair function of parkin through direct post-translational modification and/or alteration of parkin solubility (Chung et al., 2004; Yao et al., 2004; LaVoie et al., 2005, Wang et al., 2005). The molecular mechanisms underlying impairment of parkin function by these stressors are unknown. Nor is it clear whether these modifications play a role in common, sporadic forms of PD.

$\mathrm{c}$-Abl is a tightly regulated non-receptor protein tyrosine kinase involved in a wide range of cellular processes, including growth, survival and stress response (Hantschel and SupertiFurga, 2004). c-Abl is structurally homologous to the Src family of kinases in its $\mathrm{N}$-terminal region, with three distinct domains$\mathrm{SH} 3, \mathrm{SH} 2$, and a tyrosine kinase catalytic domain (Smith and 
Mayer, 2002). c-Abl and its close relative, Abl-related gene (Arg) tyrosine kinase, have long unique $\mathrm{C}$-terminal extensions that display numerous functionalities (Smith and Mayer, 2002). c-Abl shuttles between cytoplasm and nucleus and its subcellular localization determines its function in response to diverse types of stress (Van Etten, 1999). The cytoplasmic form of c-Abl is activated in cellular response to oxidative stress (Sun et al., 2000). Since oxidative stress is a prominent feature of sporadic PD (Jenner and Olanow, 1998), we investigated whether c-Abl could play pathogenic role in PD.

\section{Materials and Methods}

Plasmids. Parkin, ubiquitin, AIMP-2, FBP-1, and c-Abl constructs have been previously described (Sun et al., 2000; Zhang et al., 2000b; Chung et al., 2001; Yamamoto et al., 2005).

Cell culture. K562 human leukemic cells were cultured in RPMI 1640 containing 10\% FBS. Human embryonic kidney (HEK) cells were cultured in MEM containing 10\% FBS, SH-SY5Y human neuroblastoma cells were cultured in DMEM containing 10\% FBS. SH-SY5Y cells were treated with $100 \mu \mathrm{M}$ 1-methyl-4-phenylpyridine $\left(\mathrm{MPP}^{+}\right)$or dopamine (DA) for $24 \mathrm{~h}$, or with $250 \mu \mathrm{M} \mathrm{H}_{2} \mathrm{O}_{2}$ for $1 \mathrm{~h}$ in serum-free medium. The c-Abl inhibitor STI-571 (Gleevec, imatinib mesylate; Novartis Pharma AG) was added to cells at $10 \mu \mathrm{M}$ for $6 \mathrm{~h}$ before toxin-treatment. Cells were treated with $100 \mu \mathrm{M}$ MnTBAP (Manganese (III) Tetrakis (4-Benzoic Acid) Porphyrin Chloride) (Sigma) or $1 \mathrm{~mm} N$-acetylcysteine (NAC) $24 \mathrm{~h}$ before $\mathrm{MPP}^{+}$treatment. Cells were also transfected with c-Abl siRNA or green florescent protein (GFP) siRNA $48 \mathrm{~h}$ before $\mathrm{MPP}^{+}$treatment. All transfections were done with Lipofectamine PLUS or Lipofectamine 2000 reagent according to the manufacturer's instructions (Invitrogen). Enriched mouse primary striatal neurons were grown and differentiated as directed by the supplier (Cambrex).

Glutathione S-transferase pull-down assay. Glutathione S-transferase (GST) pull-down assays were performed according to the manufacturer using glutathione-Sepharose beads (GE Healthcare).

Coimmunoprecipitation. SH-SY5Y cells were transfected with $2 \mu \mathrm{g}$ of various plasmids and coimmunoprecipitations were performed as previously described (Zhang et al., 2000b; Chung et al., 2001).

In vitro phosphorylation. GST-parkin (200 ng) was incubated with 100 ng kinase-active c-Abl, in standard in vitro kinase assays with or without STI-571 (Sun et al., 2000).

In vitro ubiquitination. GST-parkin was preincubated with kinaseactive c-Abl for $30 \mathrm{~min}$ before initiating in vitro ubiquitination. Reactions were performed at $30^{\circ} \mathrm{C}$ in $20 \mu \mathrm{l}$ of mixture containing $50 \mathrm{~mm}$ Tris $\mathrm{HCl}$, pH7.5, $2.5 \mathrm{~mm} \mathrm{MgCl}_{2}, 2 \mathrm{~mm}$ ATP, $5 \mu \mathrm{g}$ of ubiquitin (Sigma), $100 \mathrm{ng}$ of E1 (Sigma), $400 \mathrm{ng}$ of UbcH7 (Sigma), and $200 \mathrm{ng}$ of GST-parkin. For ubiquitination of FBP-1, HEK cells were transfected with hemagglutinin (HA)-FBP-1 plasmid ( $4 \mu \mathrm{g}$ ). Cells were collected after $48 \mathrm{~h}$ and radioimmunoprecipitation assay (RIPA) lysates were subjected to immunoprecipitation with anti-HA-agarose (Sigma) and washed. GST-parkin was preincubated with kinase-active (KA) c-Abl or kinase-dead (KD) c-Abl or with kinase-active c-Abl in the presence of STI-571 $(2.5 \mu \mathrm{M})$ for 30 min before initiating in vitro ubiquitination. Reactions were performed at $30^{\circ} \mathrm{C}$ by adding a $20 \mu \mathrm{l}$ of mixture of the above in vitro ubiquitination mixture. After $2 \mathrm{~h}$, the reactions were terminated with an equal volume of $1 \times$ SDS sample buffer and the products analyzed by immunoblot with anti-FLAG and anti-HA antibodies (Sigma).

Parkin knockdown. SH-SY5Y cells were infected with lenti-shRNAparkin or lenti-shRNA-GFP $48 \mathrm{~h}$ before $\mathrm{MPP}^{+}$treatment. Cells were harvested and lysed in RIPA buffer for biochemical analysis or stained for cell viability $24 \mathrm{~h}$ after $\mathrm{MPP}^{+}$treatment. At $48 \mathrm{~h}$, knockdown efficiency of parkin shRNA was $\sim 65 \%$. STI-571 was added at $10 \mu \mathrm{M}$ for $6 \mathrm{~h}$ before $\mathrm{MPP}^{+}$treatment. To determine the toxic effects of this treatment, $\mathrm{SH}-$ SY5Y cells cultured in 6-well plates at $0.5 \times 10^{6}$ cells/well were infected as before, then $24 \mathrm{~h}$ later, treated with $100 \mu \mathrm{M} \mathrm{MPP}{ }^{+}$for $24 \mathrm{~h}$. In some cases, $10 \mu \mathrm{M}$ STI-571 was added to $6 \mathrm{~h}$ before $\mathrm{MPP}^{+}$treatment. Cells were stained with Hoechst (Invitrogen) and propidium iodide (PI) (Sigma). Infection efficiencies were determined by counting number of GFPpositive cells among Hoechst-stained cells $48 \mathrm{~h}$ postinfection. Cell death was assayed by counting PI-positive (red) cells among GFP-positive (green) cells in four randomly chosen fields in each well. These experiments were repeated three times. Average \pm SE was plotted as percentage cell death.

Human tissue. Human brain tissue was obtained through the brain donation program of the Morris K. Udall Parkinson's Disease Research Center at Johns Hopkins Medical Institutions in keeping with HIPAA (Health Insurance Portability and Accountability Act) regulations. This research proposal involves anonymous autopsy material and follows Federal Register 46.101 exemption number 4. Triton X-100 (TX-100)soluble and TX-100-insoluble fractions were collected, analyzed by immunoblot and densitometric analyses of protein bands using an Alpha Imager 2000 (Alpha Innotech). Relative levels of phospho-parkin (ratio of phospho-parkin to IP parkin), AIMP2 (ratio of AIMP2 to actin), and phospho-c-Abl (ratio of phospho-c-Abl to c-Abl, after each was normalized to actin) were expressed as mean $\pm \mathrm{SE}$. The degree of association between phospho-parkin and AIMP2 or phospho-c-Abl was calculated by comparing the normalized values using the correlation (CORREL) function in Excel.

OxyBlot analysis. Cell lysates $(50 \mu \mathrm{g})$ from postmortem samples of striatum or cortex of PD patients or age-matched controls were derivatized with 2,4-dinitrophenylhydrazine as per the manufacturer's protocol (OxyBlot, Millipore).

Neurotoxin injections in mice. All animal procedures were approved by and conformed to guidelines of Institutional Animal Care Committee (National Center for Toxicological Research, Jefferson, AR). Adult male C57BL/ 6 mice ( $n=5$ per group) were pretreated for 1 week with daily 10 $\mathrm{mg} / \mathrm{kg}$ STI-571 or vehicle alone (25\% DMSO/PBS) via intraperitoneal injection. On day 7 animals received four intraperitoneal injections of the neurotoxin, 1-methyl-4-phenyl-1,2,3,6-tetrahydropyridine (MPTP-HCl; $20 \mathrm{mg} / \mathrm{kg}$ free base; Research Biochemicals) in saline or saline alone at $2 \mathrm{~h}$ intervals. Daily STI-571 injections continued up to one more week after the last injection of MPTP. Animals were processed and prepared for biochemical and neurochemical assessment as previously described (Przedborski et al., 1996; Thomas et al., 2007).

\section{Results}

\section{Parkin is tyrosine-phosphorylated by c-Abl, blocking its E3 ubiquitin ligase activity}

GST-pull down of K562 cell lysates with GST-tagged full-length or truncated ( $\mathrm{N}$ or $\mathrm{C}$ terminal) forms of parkin (supplemental Fig. $1 a$, available at www.jneurosci.org as supplemental material) revealed that $\mathrm{N}$-terminal domain of parkin interacts with $\mathrm{c}-\mathrm{Abl}$ (Fig. 1a). Pull-down with GST-tagged proteins of full-length c-Abl, and SH3, SH2, SH2-TK (tyrosine kinase), TK-DNA binding (DBD), DBD, and F-actin domains of c-Abl (supplemental Fig. $1 b$, available at www.jneurosci.org as supplemental material) and lysates expressing FLAG-parkin showed a strong interaction of parkin with full-length c-Abl, and modest interaction with its truncated SH3 and SH2 domains (Fig. 1b). Parkin-Abl interaction is specific, since FLAG-parkin failed to interact with c-Ablrelated gene (Arg) tyrosine kinase (Fig. 1c).

In vitro $\mathrm{c}$-Abl kinase assay with myc-tagged constructs (supplemental Fig. $1 c$, available at www.jneurosci.org as supplemental material) of parkin indicated that c-Abl tyrosine phosphorylates only full-length parkin and a construct lacking the ubiquitin-like domain ( $\triangle \mathrm{UBL}$ ) (Fig. $1 d$ ), suggesting that Y143 is substrate for c-Abl. In vitro kinase reactions of GST-fusion proteins of wildtype parkin, Y143F mutant parkin, ParN and ParC with a $32 \mathrm{kDa}$ active tyrosine kinase domain of c-Abl (TK-c-Abl) (supplemental Fig. $1 d$, available at www.jneurosci.org as supplemental material) revealed increased tyrosine phosphorylation of wild-type parkin and ParN, but not of Y143F mutant parkin or ParC (Fig. 1e). STI-571, a selective c-Abl inhibitor, substantially reduced c-Ablmediated tyrosine phosphorylation of GST-parkin (Fig. 1f). Moreover, parkin phosphorylation was not observed in the ab- 
a

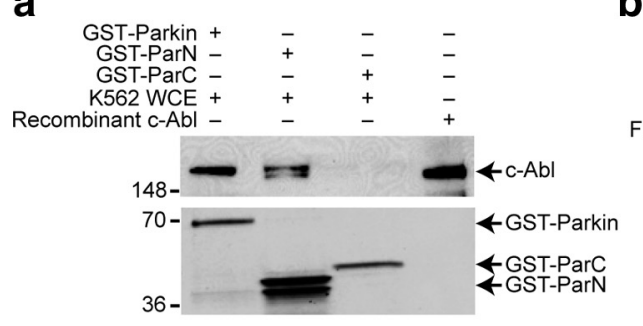

d

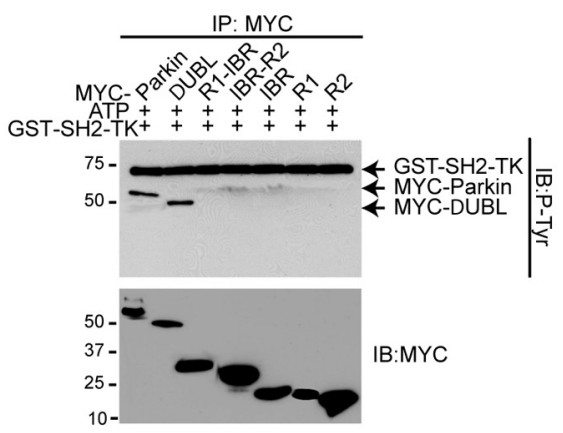

g

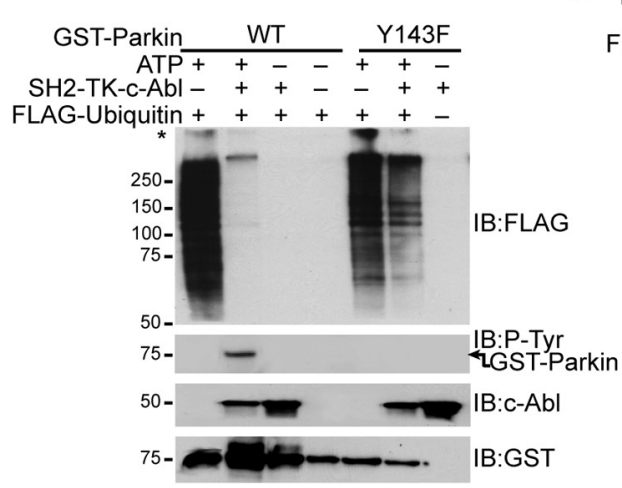

b

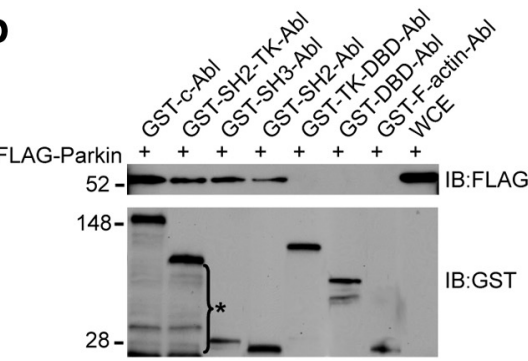

e

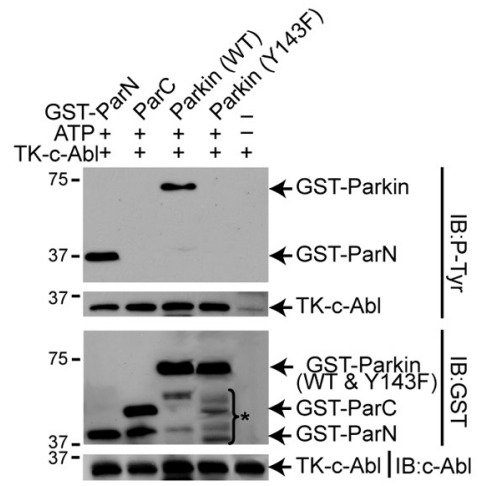

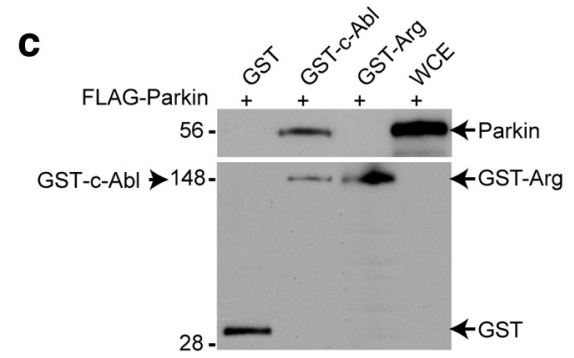

f

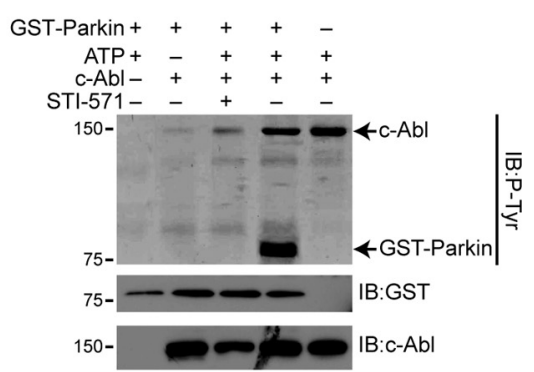

i

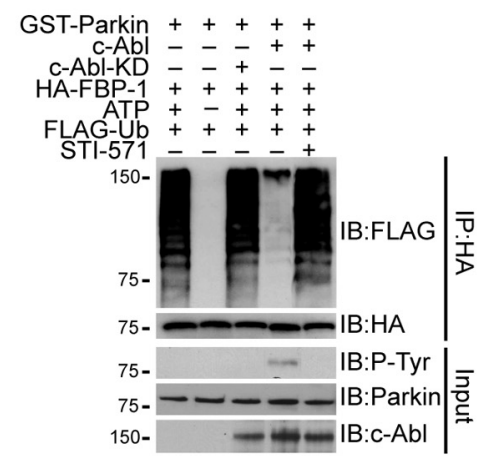

Figure 1. $\quad \boldsymbol{a}$, GST pull-down assay using GST-Parkin and parkin-fragments, GST-ParN and GST-ParC ( $\sim 1 \mu \mathrm{g}$ each) with K562 whole-cell extracts (WCE) (5 mg). $\boldsymbol{b}$, GST pull-down assay using GST-c-Abl and c-Abl fragments $(\sim 1 \mu \mathrm{g})$ as shown in c and WCE $(5 \mathrm{mg})$ of SH-SY5Y cells transfected with FLAG-parkin. *Degradation products of GST-c-Abl. c, GST pull-down assay using GST-Arg and GST-c-Abl $(\sim 1 \mu \mathrm{g})$ and WCE (5 mg) of SH-SY5Y cells transfected with FLAG-parkin. $d$, Immunoblots with anti-p-tyrosine (P-Tyr) and myc after an in vitro kinase assay using GST-SH2-TK (c-Abl) and myc immunoprecipitates from SH-SY5Y cells transfected with myc-tagged full-length and deletion mutants of parkin $(2 \mu \mathrm{g}) . e$, Immunoblots with antibodies to $P$-Tyr, GST, c-Abl after an in vitro kinase assay using TK-c-Abl and GST-ParN, GST-ParC or GST-Parkin, wild-type or Y143F parkin. *Degradation products of GST-constructs. $f$, Immunoblots with antibodies to $P$-Tyr, GST, c-Abl after an in vitro kinase assay using GST-Parkin with full-length human recombinant His-c-Abl $(100 \mathrm{ng})$ with or without STI-571 $(2.5 \mu \mathrm{M}) . \mathbf{g}$, Immunoblots of in vitro auto-ubiquitination assays using SH2-TK-Abl ( $\sim 200 \mathrm{ng}$ ) and GST-Parkin (wild-type or Y143F). * Nonspecific ubiquitination. $\boldsymbol{h}$, Immunoblot analysis of immunoprecipitates with FLAG and myc antibodies of SH-SY5Y cell lysates transfected with FLAG-tagged AIMP2 $(2 \mu \mathrm{g})$, myc-tagged WT parkin (1 $\mu \mathrm{g})$, HA-tagged ubiquitin $(1 \mu \mathrm{g})$ and GFP-tagged c-Abl-KA (1 $\mu \mathrm{g})$, incubated with $10 \mu \mathrm{m}$ STl-571 for $12 \mathrm{~h}$. $i$, Immunoblots of an in vitro GST-parkin ubiquitination assay of immunoprecipitated HA-FBP-1 with c-Abl or c-AbI KD ( $200 \mathrm{ng})$ with or without STl-571 (2.5 $\mu \mathrm{M})$. All experiments were repeated at least three times. Representative examples are presented.

sence of c-Abl (Fig. $1 f$ ). These results indicate that parkin specifically interacts with c-Abl and that parkin is phosphorylated by c-Abl at its $\mathrm{N}$-terminal domain on Y143.

In vitro ubiquitination assays using recombinant GST-parkin (wild-type or Y143F) and SH2-TK-c-Abl revealed that c-Ablmediated parkin phosphorylation substantially inhibited its E3 ubiquitin ligase activity, as demonstrated by reduced parkin autoubiquitination (Fig. 1g). The phosphorylation-resistant Y143F mutant of parkin showed little effect on auto-ubiquitination (Fig. $1 g$ ). Parkin-mediated ubiquitination of AIMP2 was reduced in the presence of c-Abl, an effect that was blocked by STI-571 (Fig. 1h). Parallel results were obtained using an alternative parkin substrate FBP-1 (Fig. 1i). Thus, parkin-mediated E3 ubiquitin ligase activity is inhibited by c-Abl-mediated phosphorylation of parkin on Y143.

\section{Loss of parkin function after oxidative stress-induced} activation of $\mathrm{c}-\mathrm{Abl}$

Cellular stress induced by $100 \mu \mathrm{M} \mathrm{MPP}{ }^{+}, 250 \mu \mathrm{M} \mathrm{H}_{2} \mathrm{O}_{2}$, or 100 $\mu \mathrm{M}$ DA activated c-Abl in SH-SY5Y cells, as measured by phospho-c-Abl levels (autophosphorylation of Y245) (Fig. 2a). Substantial parkin phosphorylation and AIMP2 accumulation was also observed (Fig. 2a). STI-571 prevented parkin phosphorylation and AIMP2 accumulation (Fig. 2a). Pretreatment of cells with superoxide dismutase mimetic MnTBAP $(100 \mu \mathrm{M})$ or antioxidant NAC ( $1 \mathrm{mM}$ ) for $24 \mathrm{~h}$ before $\mathrm{MPP}^{+}$exposure prevented parkin phosphorylation and AIMP2 accumulation (Fig. 2b). $\mathrm{MPP}^{+}$treatment also led to STI-571-inhibitable activation of c-Abl, parkin phosphorylation, and AIMP2 accumulation in primary striatal neurons (Fig. 2c). We also performed tyrosine hydroxylase immunostaining of primary mid-brain neurons 
a
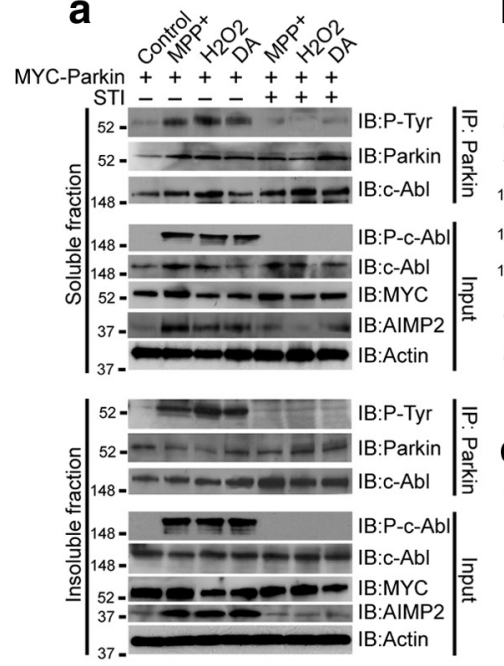

b
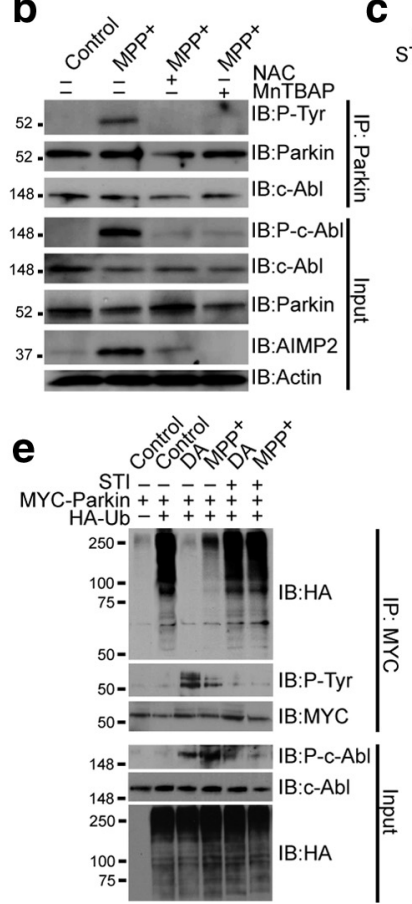

C
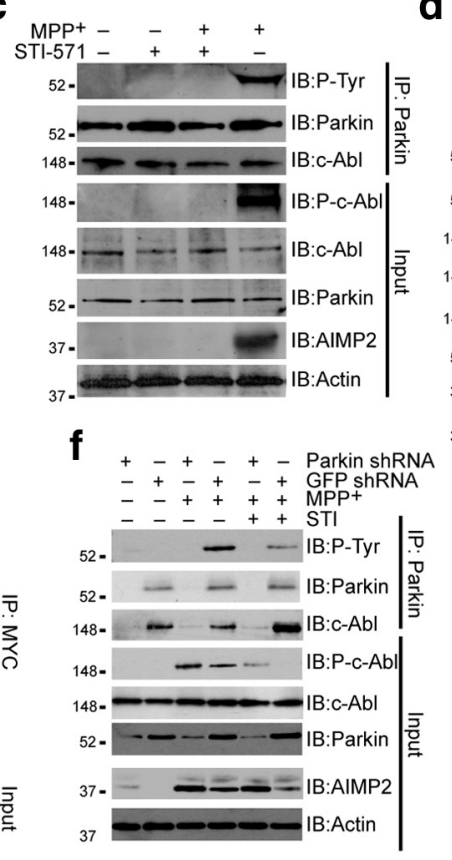

d

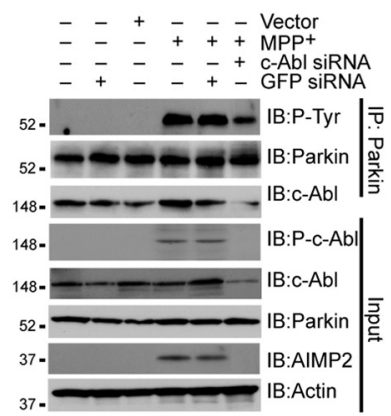

g

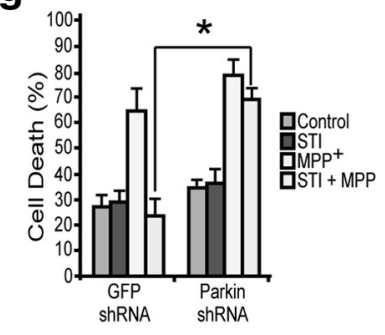

Figure 2. $\quad \boldsymbol{a}$, Immunoblots of parkin immunoprecipitates of SH-SY5Y TX-100-soluble (top) and TX-100-insoluble (bottom) fractions of cell lysates transiently transfected with myc-parkin treated with $\mathrm{MPP}^{+}(100 \mu \mathrm{M}), \mathrm{DA}(100 \mu \mathrm{M})$ for $24 \mathrm{~h}$ or with $\mathrm{H}_{2} \mathrm{O}_{2}(250 \mu \mathrm{m}$ in serum free medium) for $1 \mathrm{~h}$. In lanes 5, 6, and 7, cells were pretreated with STI-571 (STI) at $10 \mu \mathrm{m}$ for $6 \mathrm{~h}$ before exposure to toxins. $\boldsymbol{b}$, Immunoblots of parkin immunoprecipitates of SH-SY5Y cell lysates transiently transfected with myc-parkin treated with MPP ${ }^{+}(100 \mu \mathrm{m})$ for $24 \mathrm{~h}$. In lanes 3 and 4 , cells were pretreated with the superoxide dismutase mimetic MnTBAP $(100 \mu \mathrm{m})$ and antioxidant NAC $(1 \mathrm{~mm})$ for $24 \mathrm{~h}$ before exposure to MPP ${ }^{+}$.c, Immunoblots of parkin immunoprecipitates of mouse primary striatal neurons (95\% glia-free) treated with $100 \mathrm{~nm} \mathrm{MPP}{ }^{+}$for $24 \mathrm{~h}$ with or without STI-571 (2.5 $\mu \mathrm{m}$ for $6 \mathrm{~h}$ before MPP ${ }^{+}$treatment). $\boldsymbol{d}$, Immunoblots of parkin immunoprecipitates of SH-SY5Y cells transiently transfected with c-Abl siRNA or GFP siRNA $48 \mathrm{~h}$ before MPP ${ }^{+}(100 \mu \mathrm{M})$ treatment. $\boldsymbol{e}$, Immunoblots of myc immunoprecipitates of SH-SY5Y cell lysates transiently transfected with myc-parkin and HA-ubiquitin and treated with MPP ${ }^{+}$or DA $(100 \mu \mathrm{M})$ for $24 \mathrm{~h}$. In lanes 5 and 6 , cells were pretreated with STI- 571 at $10 \mu \mathrm{m}$ for $6 \mathrm{~h}$ before exposure to MPP ${ }^{+}$or DA.f Immunoblots of parkin immunoprecipitates of SH-SY5Y cells, infected with either lenti-shRNA to parkin or lenti-shRNA to GFP, treated with $100 \mu \mathrm{M}$ MPP ${ }^{+}$. Some samples were incubated with $10 \mu \mathrm{M}$ STI-571 for $6 \mathrm{~h}$ before MPP ${ }^{+}$treatment. $\boldsymbol{g}$, Cell death plotted as percentage of PI-positive cells among GFP-positive cells of SH-SY5Y cells infected with lenti-shRNA-parkin or lenti-shRNA-GFP alone $48 \mathrm{~h}$ before treatment with MPP ${ }^{+}(100 \mu \mathrm{M})$. Some samples were incubated with $10 \mu \mathrm{M} \mathrm{STI-571} \mathrm{for} 6 \mathrm{~h}$ before MPP ${ }^{+}$treatment. ${ }^{*} p<0.05$. Differences among means were analyzed using one-way ANOVA with the different treatments as the independent factor followed by Newman-Keuls post hoc analysis. All experiments were repeated at least three times. Representative examples are presented.

treated with $\mathrm{MPP}^{+}$with or without STI-571. Loss of $\mathrm{TH}^{+}$immunostaining and damage to neuronal morphology was observed in $\mathrm{MPP}^{+}$groups which was significantly reversed by STI-571 (supplemental Fig. 2, available at www.jneurosci.org as supplemental material). $\mathrm{MPP}^{+}(100 \mathrm{nM}, 24 \mathrm{~h})$ failed to activate $\mathrm{c}-\mathrm{Abl}$ in pure astrocytes (data not shown), suggesting that this pathway is specific to neurons. Also, we could not detect an active c-Abl signal in astrocytes (supplemental Fig. 3, available at www. jneurosci.org as supplemental material). Knockdown of c-Abl by siRNA prevented $\mathrm{MPP}^{+}$-induced c-Abl activation, parkin phosphorylation and AIMP2 accumulation, whereas control vector or GFP siRNA had no effect (Fig. $2 d$ ). $\mathrm{MPP}^{+}$and DA substantially reduced parkin's E3-ligase activity, an effect that was blocked by STI-571 pretreatment (Fig. 2e).

To ascertain whether the protective effect of STI-571 requires parkin, its ability to protect against $\mathrm{MPP}^{+}$was monitored in cells with parkin-knockdown. Parkin knockdown disrupted c-Abl/ parkin interaction and reduced STI-571 ability to prevent AIMP2 accumulation after MPP ${ }^{+}$treatment (Fig. 2f). STI-571 rescue of $\mathrm{MPP}^{+}$-induced cell death was prevented by parkin knockdown (Fig. $2 g$ ). Thus, parkin is indeed required for the protective effects of STI-571.

\section{Parkin is tyrosine-phosphorylated in the striatum of PD patients}

To determine potential relevance of c-Abl-mediated parkin phosphorylation to PD pathology, we investigated presence of tyrosine-phosphorylated parkin in postmortem brain tissue prepared from striatum, cingulate cortex, and cerebellum from PD patients and age-matched controls (supplemental Table 1, available at www.jneurosci.org as supplemental material). There was a threefold increase in tyrosine-phosphorylated parkin in soluble fraction of striatal tissue of PD patients compared with controls (Fig. 3a,b). Binding of parkin to c-Abl was increased in PD patients compared with controls (Fig. $3 a, b$ ). In addition, a fourfold increase in AIMP2, 3-fold increase in FBP-1, and 2.5-fold increase in phospho-c-Abl were observed in $\mathrm{PD}$ striatal lysates, with no change in the levels of c-Abl itself (Fig. 3a,b). A significant positive correlation was observed between phospho-parkin and phospho-c-Abl $(r=0.8, p<0.05)$, FBP-1 $(r=0.7, p<0.05)$, and AIMP2 $(r=0.514, p<0.05)$ in soluble fraction of striatum. Similarly, a twofold increase in tyrosine-phosphorylated parkin, as well as high levels of parkin, a twofold increase in AIMP2, and a threefold increase in FBP-1 were observed in the insoluble fraction of striatum from PD patients compared with controls (Fig. $3 c, d)$. Consistent with the notion that tyrosine-phosphorylation leads to parkin inactivation, levels of ubiquitinated parkin, measured by ubiquitin reactivity in immunoprecipitated parkin, were significantly lower in both soluble and insoluble fractions of PD striatum samples (Fig. $3 a, c$ ).

Tyrosine phosphorylation of parkin was specific to nigrostriatum, as the levels of phospho-parkin, phospho-c-Abl, and AIMP2 in cortex were unaffected (Fig. $3 e, f$ ), even in cases with cortical and limbic dementia with Lewy Bodies, and in cerebel- 

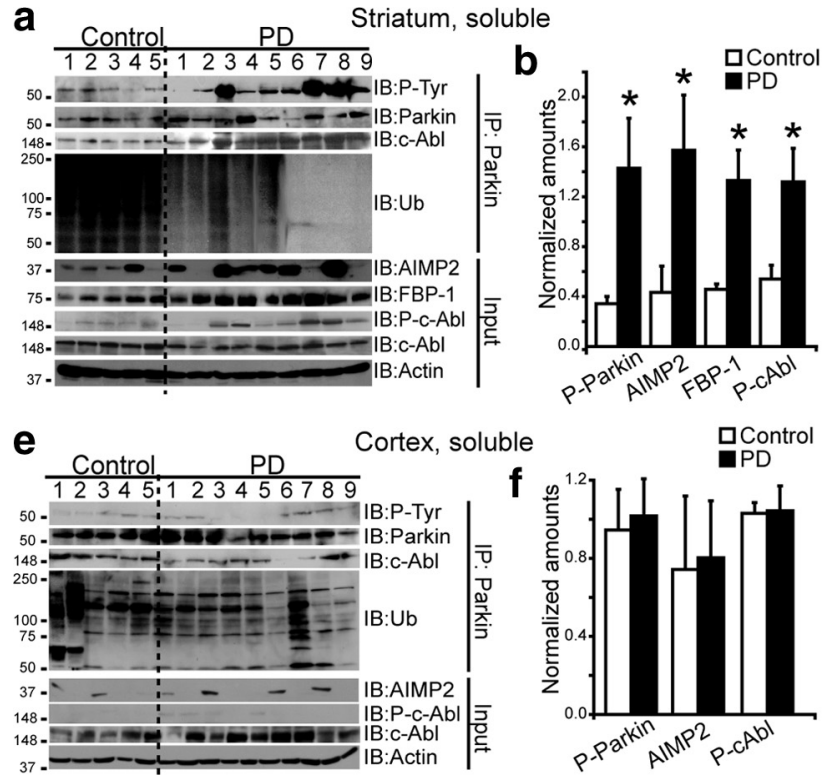
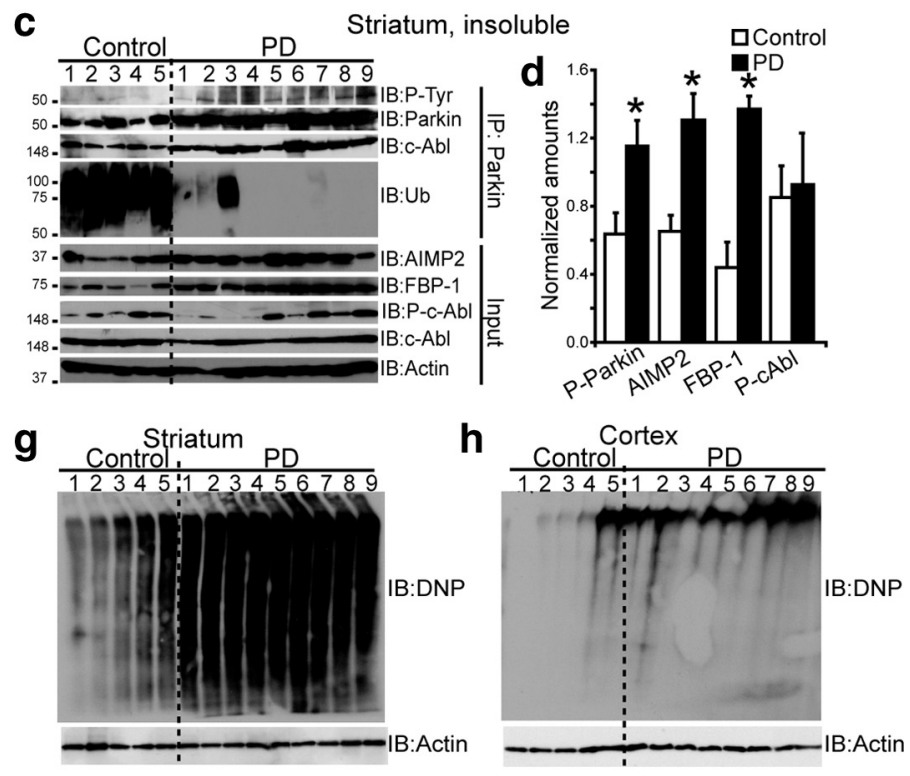

Figure 3. $\boldsymbol{a}, \boldsymbol{c}, \boldsymbol{e}$, Immunoblots of parkin immunoprecipitates $(2 \mathrm{mg})$ prepared from lysates of TX-100-soluble striatum (a), TX-100-insoluble striatum (c), and TX-100-soluble cortex lysates $(\boldsymbol{e})$. Brain tissue lysates $(100 \mu \mathrm{g})$ were immunoblotted with antibodies to parkin, AIMP2, FBP-1, ubiquitin (Ub), P-tyr, P-c-Abl (p-Y245), c-Abl, and actin. $\boldsymbol{b}, \boldsymbol{d}, \boldsymbol{f}$, Ratios of phospho-parkin/IP parkin and AIMP2/actin from lysates of TX-100-soluble striatum (b), TX-100-insoluble striatum (d), and TX-100-soluble cortex for control versus PD (f) $\left({ }^{*} p<0.05\right.$, Student's $t$ test). $\boldsymbol{g}, \boldsymbol{h}, 0$ xyBlot analysis of proteins from postmortem striatum $(\boldsymbol{g})$, and cortex of PD patients and age-matched controls $(\boldsymbol{h})$. All experiments were repeated at least three times. Representative examples are presented.

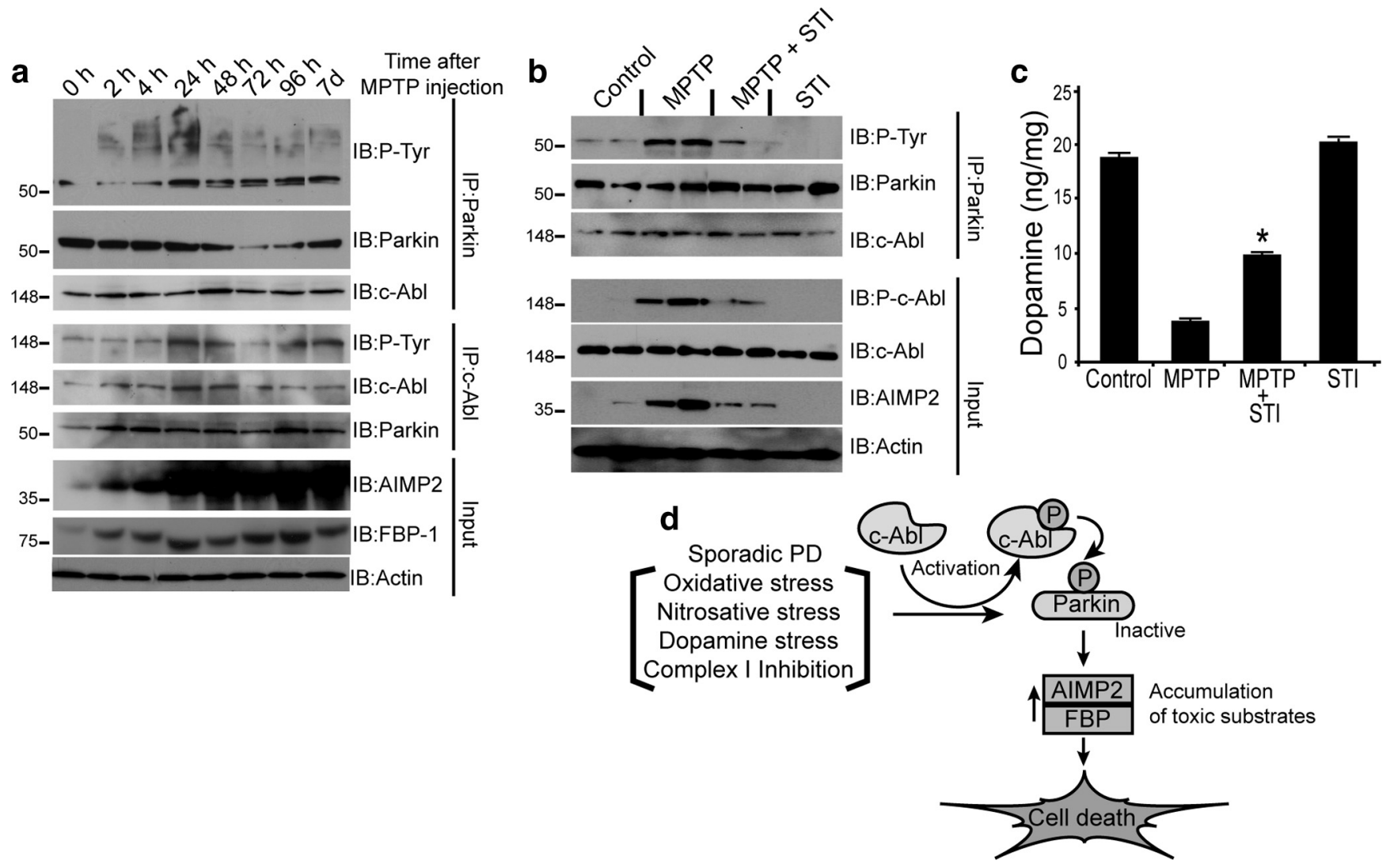

Figure 4. $\quad \boldsymbol{a}$, Immunoblots of c-Abl and parkin IP samples $(2 \mathrm{mg})$ from striatum tissue lysates of saline- or MPTP-treated mice (4 doses of $20 \mathrm{mg} / \mathrm{kg}$ MPTP, i.p., at $2 \mathrm{~h}$ intervals at indicated time points). $\boldsymbol{b}$, Immunoblots of parkin immunoprecipitates of striatum lysates $(100 \mu \mathrm{g}) 7 \mathrm{~d}$ after MPTP treatment with or without STI-571(STI) (10 mg/kg, i.p.), (daily injection for $7 \mathrm{~d}$ before and $7 \mathrm{~d}$ after MPTP injection) compared with saline- and STI-injected control mice. c, STI-571 prevents MPTP-induced depletion of striatal dopamine in mice, as assessed by HPLC/electrochemical detection. Each value is mean \pm SEM derived from 10 animals/group.Statistical significance was assessed using one-way ANOVA and Student-Newman-Keuls multiple-comparisons test. ${ }^{*} p<0.05$ for difference from MPTP group. $\boldsymbol{d}$, Schematic representation of the pathway by which parkin tyrosine phosphorylation can lead to cell death and PD. Oxidative or dopamine stress from an external source or generated during sporadic PD activates c-Abl. Activated c-Abl tyrosine phosphorylates parkin resulting in loss of ubiquitin ligase activity, leading to accumulation of toxic parkin substrates and neuronal death. 
lum (data not shown), which is largely unaffected in PD. We were unable to detect FBP-1 in cortex reliably. OxyBlot analysis of striata of PD patients showed a prominent pattern of oxidized proteins compared with controls (Fig. $3 g, h$ ). In addition, the oxidation profile was severalfold higher in striatum than in cortex of PD patients, perhaps accounting for the preferential parkin phosphorylation and accumulation of its substrates in the nigrostriatum.

\section{Inhibition of c-Abl protects against MPTP-induced nigrostriatal toxicity}

Treatment of mice with the potent parkinsonian neurotoxin, MPTP (Dauer and Przedborski, 2003) (4 doses of $20 \mathrm{mg} / \mathrm{kg}$, i.p., every $2 \mathrm{~h}$ ) led to substantial c-Abl activation $24 \mathrm{~h}$ after the last dose of MPTP, as indicated by increased striatal levels of phospho-c-Abl, tyrosine phospho-parkin, AIMP2, and FBP-1, sustained for up to $7 \mathrm{~d}$ (Fig. $4 a$ ). STI-571 (10 mg/kg, i.p., daily for 1 week before and 1 week after MPTP) treatment resulted in protection against MPTP-induced injury, as reflected by significant decreases in levels of phospho-c-Abl, phospho-parkin, and AIMP2 (Fig. 4b). Moreover, the MPTP-induced loss of striatal dopamine was partially mitigated by STI-571 treatment (Fig. 4c). These results suggest that activation of c-Abl contributes to neurotoxic effects of MPTP through inhibitory tyrosine phosphorylation of parkin.

\section{Discussion}

Here we report our novel observation that parkin interacts with and is phosphorylated at tyrosine-143 by c-Abl. Activation of $\mathrm{c}$-Abl and parkin tyrosine phosphorylation occur after oxidative and dopamine stress both in vitro and in vivo, causing significant loss of parkin's ubiquitin E3-ligase activity and leading to accumulation of neurotoxic AIMP2 and FBP-1, ultimately compromising parkin's protective function (see scheme, Fig. 4d). STI-571, a selective c-Abl inhibitor, prevented parkin tyrosine phosphorylation, preserved its E3-ligase activity and cytoprotective function. The protective effect of STI-571 was parkin-dependent, since shRNA knockdown of parkin specifically attenuated STI-571 protection. Moreover, we observed tyrosine phosphorylation of c-Abl and parkin, along with accumulation of toxic parkin substrates, AIMP2 (p38/JTV-1) and FBP-1, in nigrostriatum of PD patients. There was significant correlation among tyrosine-phosphorylated parkin, activated c-Abl, and AIMP2 and FBP-1 levels in striatum of PD patients. These data provide convincing evidence for a novel oxidative stressinduced cell signaling pathway that negatively regulates parkin function through c-Abl-mediated tyrosine phosphorylation and may contribute to nigrostriatal neuronal injury and disease progression in sporadic PD.

Recently, it has been reported that oxidative (Wang et al., 2005), nitrosative (Chung et al., 2004; Yao et al., 2004), and dopaminergic (LaVoie et al., 2005) stress impair parkin function by direct modification and/or through alteration in parkin solubility, thus linking parkin to sporadic PD. However, the mechanisms underlying parkin inactivation have remained unclear. Our data provide a molecular mechanism for parkin inactivation, and support a role of parkin in pathogenesis of more common sporadic form of PD. Thus, oxidative and dopamine-stress lead to c-Abl activation, parkin tyrosine phosphorylation and the consequent loss of parkin ubiquitination-dependent cytoprotective function. c-Abl-mediated parkin inactivation in response to oxidative and dopaminergic stress seems to be the dominant pathway induced by these stressors, since the c-Abl inhibitor, STI-571, blocked inactivation of parkin.
Attempts to characterize tyrosine phosphorylation of parkin by capillary HPLC electrospray tandem mass spectrometry (HPLC-ESI-MS/MS) both in vitro and in vivo were unsuccessful, despite the ability to detect the nonphosphorylated peptide in both the precursor and targeted product scans (data not shown). We suspect that detection of Y143 phospho-peptide via MS/MS is not technically feasible because of poor solubility, since parkin peptides containing phosphorylated Y143 failed to dissolve in solvents used in the MS/MS analysis (data not shown). Since we were unable to prove definitively via mass-spectrometry that parkin is tyrosine-phosphorylated at Y143, we cannot exclude the possibility that there are additional c-Abl targets that may contribute to the pathogenesis of PD.

Our finding that this pathway is seen predominantly in the striatum suggests that dopamine-containing cells of the nigrostriatum are particularly predisposed. $\mathrm{c}-\mathrm{Abl}$ activation and parkin tyrosine phosphorylation appear to reflect processes that are unique to nigrostriatum and not necessarily associated with inclusion bodies, since we did not observe c-Abl activation and tyrosine phosphorylation of parkin in the cortex, even in the four PD patients with neocortical Lewy bodies. Furthermore, parkin tyrosine phosphorylation and AIMP2 accumulation in striatum compared with cortex appears to be associated with increased oxidative stress in the striatum of PD patients, as indicated by OxyBlot analysis. Since oxidative stress is intimately involved in sporadic $\mathrm{PD}$, we propose a novel stress-induced cell signaling mechanism featuring activated c-Abl, which inhibits parkin function and consequently increases cell death as a result of accumulation of cytotoxic parkin substrates, such as AIMP2 (Fig. 4d).

The c-Abl inhibitor STI-571 is widely used chemotherapeutic agent for chronic myelogenous leukemia. The finding that STI571 inhibits c-Abl's deleterious effects on parkin by preventing its phosphorylation and preserving its protective function, holds promise for further testing of this agent as a neuroprotective therapeutic for PD. Since STI-571 has limited brain bioavailability (Breedveld et al., 2005), the amount of protection afforded by inhibition of c-Abl in vivo may be greatly improved by using related compounds with enhanced brain penetration. The identification of c-Abl tyrosine phosphorylation-mediated inhibition of parkin activity and its pathological relevance as demonstrated in PD will pave the way for better understanding of the pathophysiology of this disease.

\section{References}

Braak H, Del Tredici K, Rüb U, de Vos RA, Jansen Steur EN, Braak E (2003) Staging of brain pathology related to sporadic Parkinson's disease. Neurobiol Aging 24:197-211.

Breedveld P, Pluim D, Cipriani G, Wielinga P, van Tellingen O, Schinkel AH, Schellens JH (2005) The effect of Bcrp1 (Abcg2) on the in vivo pharmacokinetics and brain penetration of imatinib mesylate (Gleevec): implications for the use of breast cancer resistance protein and P-glycoprotein inhibitors to enable the brain penetration of imatinib in patients. Cancer Res 65:2577-2582.

Chung KK, Zhang Y, Lim KL, Tanaka Y, Huang H, Gao J, Ross CA, Dawson VL, Dawson TM (2001) Parkin ubiquitinates the alpha-synucleininteracting protein, synphilin-1: implications for Lewy-body formation in Parkinson disease. Nat Med 7:1144-1150.

Chung KK, Thomas B, Li X, Pletnikova O, Troncoso JC, Marsh L, Dawson VL, Dawson TM (2004) S-nitrosylation of parkin regulates ubiquitination and compromises parkin's protective function. Science 304:1328-1331.

Corti O, Hampe C, Koutnikova H, Darios F, Jacquier S, Prigent A, Robinson JC, Pradier L, Ruberg M, Mirande M, Hirsch E, Rooney T, Fournier A, Brice A (2003) The p38 subunit of the aminoacyl-tRNA synthetase complex is a Parkin substrate: linking protein biosynthesis and neurodegeneration. Hum Mol Genet 12:1427-1437. 
Dauer W, Przedborski S (2003) Parkinson's disease: mechanisms and models. Neuron 39:889-909.

Dawson TM, Dawson VL (2003) Molecular pathways of neurodegeneration in Parkinson's disease. Science 302:819-822.

Hantschel O, Superti-Furga G (2004) Regulation of the c-Abl and Bcr-Abl tyrosine kinases. Nat Rev Mol Cell Biol 5:33-44.

Jenner P, Olanow CW (1998) Understanding cell death in Parkinson's disease. Ann Neurol 44:S72-S84.

Kitada T, Asakawa S, Hattori N, Matsumine H, Yamamura Y, Minoshima S, Yokochi M, Mizuno Y, Shimizu N (1998) Mutations in the parkin gene cause autosomal recessive juvenile parkinsonism. Nature 392:605-608.

Ko HS, von Coelln R, Sriram SR, Kim SW, Chung KK, Pletnikova O, Troncoso J, Johnson B, Saffary R, Goh EL, Song H, Park BJ, Kim MJ, Kim S, Dawson VL, Dawson TM (2005) Accumulation of the authentic parkin substrate aminoacyl-tRNA synthetase cofactor, p38/JTV-1, leads to catecholaminergic cell death. J Neurosci 25:7968-7978.

Ko HS, Kim SW, Sriram SR, Dawson VL, Dawson TM (2006) Identification of far upstream element-binding protein-1 as an authentic parkin substrate. J Biol Chem 281:16193-16196.

LaVoie MJ, Ostaszewski BL, Weihofen A, Schlossmacher MG, Selkoe DJ (2005) Dopamine covalently modifies and functionally inactivates parkin. Nat Med 11:1214-1221.

Matsuda N, Kitami T, Suzuki T, Mizuno Y, Hattori N, Tanaka K (2006) Diverse effects of pathogenic mutations of Parkin that catalyze multiple monoubiquitylation in vitro. J Biol Chem 281:3204-3209.

Moore DJ, West AB, Dawson VL, Dawson TM (2005) Molecular pathophysiology of Parkinson's disease. Annu Rev Neurosci 28:57-87.

Olanow CW, Tatton WG (1999) Etiology and pathogenesis of Parkinson's disease. Annu Rev Neurosci 22:123-144.

Przedborski S, Jackson-Lewis V, Yokoyama R, Shibata T, Dawson VL, Dawson TM (1996) Role of neuronal nitric oxide in 1-methyl-4-phenyl-1,2,3,6tetrahydropyridine (MPTP)-induced dopaminergic neurotoxicity. Proc Natl Acad Sci U S A 93:4565-4571.

Shimura H, Hattori N, Kubo S, Mizuno Y, Asakawa S, Minoshima S, Shimizu N, Iwai K, Chiba T, Tanaka K, Suzuki T (2000) Familial Parkinson disease gene product, parkin, is a ubiquitin-protein ligase. Nat Genet 25:302-305.
Smith JM, Mayer BJ (2002) Abl: mechanisms of regulation and activation. Front Biosci 7:d31-d42.

Sriram SR, Li X, Ko HS, Chung KK, Wong E, Lim KL, Dawson VL, Dawson TM (2005) Familial-associated mutations differentially disrupt the solubility, localization, binding and ubiquitination properties of parkin. Hum Mol Genet 14:2571-2586.

Sun X, Majumder P, Shioya H, Wu F, Kumar S, Weichselbaum R, Kharbanda S, Kufe D (2000) Activation of the cytoplasmic c-Abl tyrosine kinase by reactive oxygen species. J Biol Chem 275:17237-17240.

Thomas B, von Coelln R, Mandir AS, Trinkaus DB, Farah MH, Leong Lim K, Calingasan NY, Flint Beal M, Dawson VL, Dawson TM (2007) MPTP and DSP-4 susceptibility of substantia nigra and locus coeruleus catecholaminergic neurons in mice is independent of parkin activity. Neurobiol Dis 26:312-322

Tsai KK, Yuan ZM (2003) c-Abl stabilizes p73 by a phosphorylationaugmented interaction. Cancer Res 63:3418-3424.

Van Etten RA (1999) Cycling, stressed-out and nervous: cellular functions of c-Abl. Trends Cell Biol 9:179-186.

von Coelln R, Dawson VL, Dawson TM (2004) Parkin-associated Parkinson's disease. Cell Tissue Res 318:175-184.

Wang C, Ko HS, Thomas B, Tsang F, Chew KC, Tay SP, Ho MW, Lim TM, Soong TW, Pletnikova O, Troncoso J, Dawson VL, Dawson TM, Lim KL (2005) Stress-induced alterations in parkin solubility promote parkin aggregation and compromise parkin's protective function. Hum Mol Genet 14:3885-3897.

Yamamoto A, Friedlein A, Imai Y, Takahashi R, Kahle PJ, Haass C (2005) Parkin phosphorylation and modulation of its E3 ubiquitin ligase activity. J Biol Chem 280:3390-3399.

Yao D, Gu Z, Nakamura T, Shi ZQ, Ma Y, Gaston B, Palmer LA, Rockenstein EM, Zhang Z, Masliah E, Uehara T, Lipton SA (2004) Nitrosative stress linked to sporadic Parkinson's disease: $S$-nitrosylation of parkin regulates its E3 ubiquitin ligase activity. Proc Natl Acad Sci U S A 101:10810-10814.

Zhang Y, Dawson VL, Dawson TM (2000a) Oxidative stress and genetics in the pathogenesis of Parkinson's disease. Neurobiol Dis 7:240-250.

Zhang Y, Gao J, Chung KK, Huang H, Dawson VL, Dawson TM (2000b) Parkin functions as an E2-dependent ubiquitin-protein ligase and promotes the degradation of the synaptic vesicle-associated protein, CDCrel-1. Proc Natl Acad Sci U S A 97:13354-13359. 